\title{
BER based Performance Evaluation of MC-CDMA System
}

\author{
Pooja Tomar \\ UG Student \\ Department of E.C. \\ SVVV, Indore, M.P, India
}

\author{
Dilip Mandloi \\ Assistant Professor \\ Department of E.C. \\ SVVV, Indore, M.P., India
}

\begin{abstract}
Multi-Carrier Code Division Multiple Access (MC-CDMA) is one of the advanced technique which supports various multimedia services such as image, data, speech, audio and video provided by the today's diverse wireless communication systems. In this paper, we investigate the Bit Error Rate (BER) performance of MC-CDMA system with Zero Forcing (ZF) and Minimum Mean Square Error (MMSE) equalization techniques through MATLAB simulation. The simulation has been carried out using Binary Phase Shift Keying (BPSK) and Quadrature Phase Shift Keying (QPSK) modulation techniques along with $1 / 2$ rate convolution coding over frequency selective Rayleigh fading channel. Simulation results show the effects of different parameters such as the number of users, equalization techniques, modulation techniques and convolutional coding on the performance of MC-CDMA system.
\end{abstract}

\section{Keywords}

MC-CDMA, ZF, MMSE, BPSK, QPSK, Spreading codes, Convolution codes

\section{INTRODUCTION}

Multi-Carrier Code Division Multiple Access (MC-CDMA) is one of the advanced technique which provides high speed wireless communication. MC-CDMA is a combination of OFDM and CDMA thus it is robust against multipath environment and employs multiuser multiplexing services [1]. It provides a solution for the problems encountered in multipath propagation i.e. efficient utilization of bandwidth, frequency selectivity and diversity. In Wireless technology, Equalizers are used to recover signals suffering from inter symbol interference [2]. Also to reduce errors, Convolutional encoding is employed with Viterbi decoding [3].

In this paper, the performance of MCCDMA system under frequency selective Rayleigh fading channel using different modulation techniques such as BPSK and QPSK, is evaluated to investigate the effects of different parameters on the system performance, such as equalization techniques ; number of users; and convolutional coding. MC-CDMA performances can be considerably improved by proper selection of these parameters. Equalization techniques used are Zero Forcing (ZF) and Minimum Mean Square Error (MMSE) and $1 / 2$ rate convolution coding is used. The Bit Error Rate (BER) versus Signal to Noise Ratio (SNR) performance of MC-CDMA system is evaluated using MATLAB simulation.

The paper consists of 5 sections. System model is explained in section 2. Simulation parameters and results are given in section 3. Conclusion and References are in section 4 and 5 respectively.

\section{SYSTEM MODEL}

\subsection{MC-CDMA system}

MC-CDMA system model is shown in Figure 1, the no. of users considered are 2,8 and 16 with corresponding sequences of symbols. At the transmitter the following steps are implemented. The binary data of the users is encoded using $1 / 2$ rate convolution encoder then the encoded data is modulated by different modulation techniques or symbol mapping schemes i.e. BPSK and QPSK. The serial input data stream is converted into parallel streams and are spread using spreading codes in the frequency domain by multiplying them with spreading codes i.e. Walsh-Hadamard codes to distinguish between users. The spread data is mapped to $\mathrm{N}$ orthogonal subcarriers through IDFT and converted back to serial data. Finally cyclic prefix is appended to introduce the guard interval and to eliminate inter symbol interference then the signal is transmitted through channel in presence of Additive White Gaussian Noise (AWGN). The channel model considered is frequency selective Rayleigh fading channel. At the receiver side the signal is demodulated by recovering Fourier coefficients in DFT and processed signal is despread using the same respective Walsh- Hadamard codes used at the transmitter side. Demodulated signals are decoded with Viterbi algorithm which performs maximum likelihood decoding of convolutional codes.

\subsection{MC-CDMA simulation model}

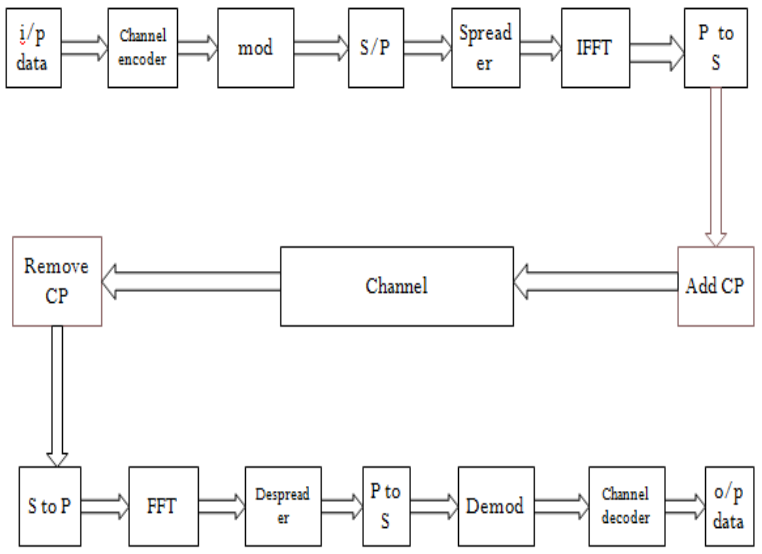

Fig 1: Simulation model of MC-CDMA system

\subsection{Convolution coding}

To reduce the bit errors introduced during the transmission of a modulated signal through a wireless channel, Convolution coding is used. One of the parameters which define Convolutional codes is the code rate. The code rate $(\mathrm{k} / \mathrm{n})$ is the 
ratio of the number of information bits fed into the convolutional encoder $(\mathrm{k})$ to the number of encoded bits generated by encoder (n) in a given encoder cycle. Using convolutional codes a continuous sequence of information bits is mapped into a continuous sequence of encoder output bits. The encoded bits depend not only on current $\mathrm{k}$ input bits but also on past input bits [4]. The Viterbi decoding strategy is mainly used to decode convolutional codes.

\subsection{Spreading codes}

Walsh-Hadamard sequences are bipolar spreading sequences which are used for channel separation in DS-CDMA. They are mathematically orthogonal codes with zero cross correlation in ideal conditions and can be generated easily [5]. When two Walsh-Hadamard codes are correlated, the result is intelligible only if these two codes are the same. A basic Hadamard matrix is given by $\begin{array}{cc}1 & 1 \\ 1 & -1\end{array}$. An $\mathrm{N} \mathrm{x} \mathrm{N} \mathrm{matrix} \mathrm{is} \mathrm{built} \mathrm{from} \mathrm{this}$ basic matrix by repetition using the formula: $\begin{array}{cc}H_{N} & H_{N} \\ H_{N} & \overline{H_{N}}\end{array}$

\subsection{Combining Techniques}

Equalizers combats inter symbol interference. At the receiver side, the signals from various paths are combined by combining techniques or equalization techniques to reduce ISI. The Equalization techniques are as follows:

\subsubsection{Zero Forcing $(Z F)$}

Zero forcing equalization is a technique of linear equalization algorithm. ZF applies channel inversion and can eliminate multiple access interference by restoring the orthogonality between the spread data in the downlink [6]. The zero forcing equalizer $\mathrm{C}(\mathrm{f})$ for a channel with frequency response $\mathrm{F}(\mathrm{f}))$ is constructed by $C(f)=1 / F(f)$. Thus the combination of channel and equalizer gives a flat frequency response and linear phase $\mathrm{C}(\mathrm{f}) \mathrm{F}(\mathrm{f})=1$. This equalizer forces ISI to zero but can enhance noise power. $\mathrm{Pe}=1 / 2\left[1-\left(\left(\mathrm{E}_{\mathrm{b}} / \mathrm{N}_{0}\right) /\left(\left(\mathrm{E}_{\mathrm{b}} / \mathrm{N}_{0}\right)+1\right)\right) 1 / 2\right]$ gives the probability of error [2].

\subsubsection{Minimum mean square error (MMSE)}

MMSE minimizes the total power of the noise and ISI components in the output unlike ZF and it may not eliminate ISI completely [6]. It works on MSE criteria and calculates the mean square error for the fitted values of dependant variables. If ' $a$ ' is an unknown variable which depends on a known variable ' $b$ ', an estimator $a^{b}$ is a function of the measurement of ' $b$ ' and hence the mean square error is give by $\mathrm{E}\left\{\left[\mathrm{A}^{\wedge} \mathrm{B} 2\right]\right\}[2]$.

\section{SIMULATION RESULTS}

In this section, the simulation results comprising of the BER Vs SNR performance of Multicarrier CDMA system over frequency selective Rayleigh channel is presented and discussed to investigate the effects of different parameters such as combining techniques (ZF and MMSE), modulation schemes (BPSK and QPSK), number of users and convolutional coding on the performance of MC-CDMA system. Simulation is done through MATLAB. Simulation parameters are given in Table 1.
Table 1: Simulation Parameters

\begin{tabular}{|c|c|}
\hline Parameters & Value \\
\hline Number of input data bits & 10000 \\
\hline Number of sub-carriers & 16 \\
\hline Number of users & $2,8,16$ \\
\hline Channel coding & Convolutional coding \\
\hline Code rate & $1 / 2$ \\
\hline Spreading codes & Walsh-Hadamard codes \\
\hline Modulation techniques & BPSK,QPSK \\
\hline Channel model & Rayleigh \\
\hline Spreading code length $L$ & 16 \\
\hline Combining Techniques & ZF,MMSE \\
\hline
\end{tabular}

- $\quad$ Effect of combining techniques

Fig 2 shows The comparison of performance of $\mathrm{ZF}$ and MMSE combining techniques for MC-CDMA system. Here BPSK modulation is used for different no. of users i.e. 2, 8, 16.The BER Vs SNR curves shows that MMSE performs better than $\mathrm{ZF}$.

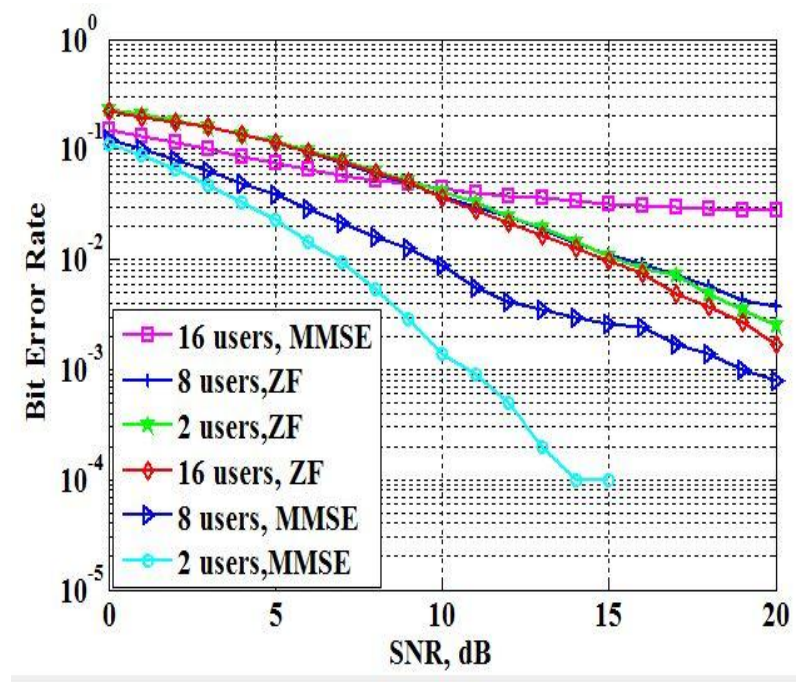

Fig 2: Comparison of ZF and MMSE

- Effect of different modulation techniques

Comparison of BPSK and QPSK modulation techniques is shown in Fig 3. BPSK performs better than QPSK. Both ZF and MMSE combining techniques are used. 


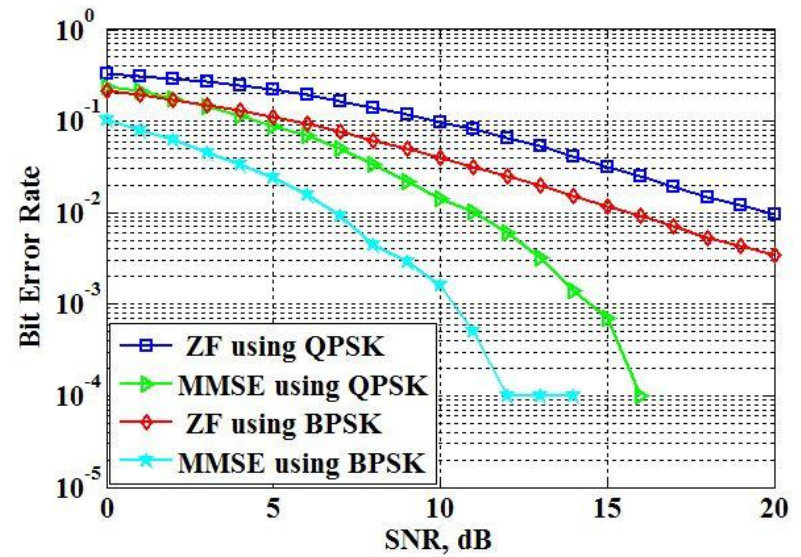

Fig 3: Comparison of BPSK and QPSK

\section{- $\quad$ Effect of number of users}

Fig 4 shows that as no. of users increases the SNR decreases and BER increases. Thus BER Vs SNR performance for less users is better than that for more users.

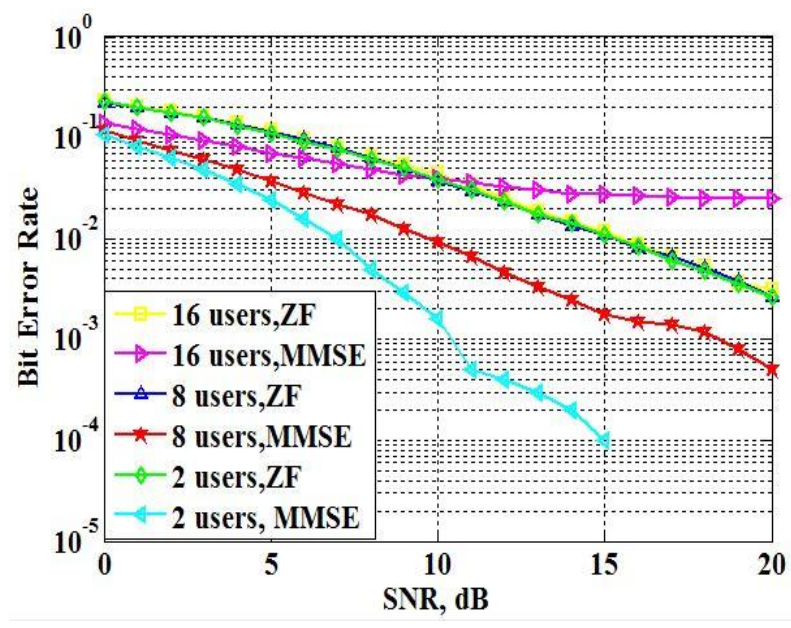

Fig 4: Comparison between performance of different no .of users

- $\quad$ Effect of convolutional coding

Fig 5, 6 shows the BER versus SNR performance comparison of MC-CDMA system with convolution codes and without convolutional codes evaluated for different no of users i.e. 2 and 8 using BPSK modulation. In Fig 5, ZF is used as combining technique and in Fig 6, MMSE is used as combining technique. Clearly, application of convolutional coding improves performance of MC-CDMA system.

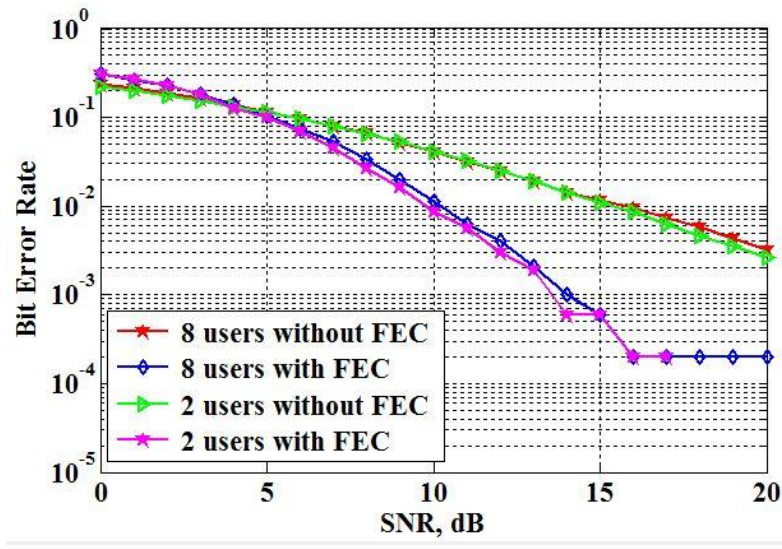

Fig 5: Simulated BER with and without FEC codes using $\mathbf{Z F}$

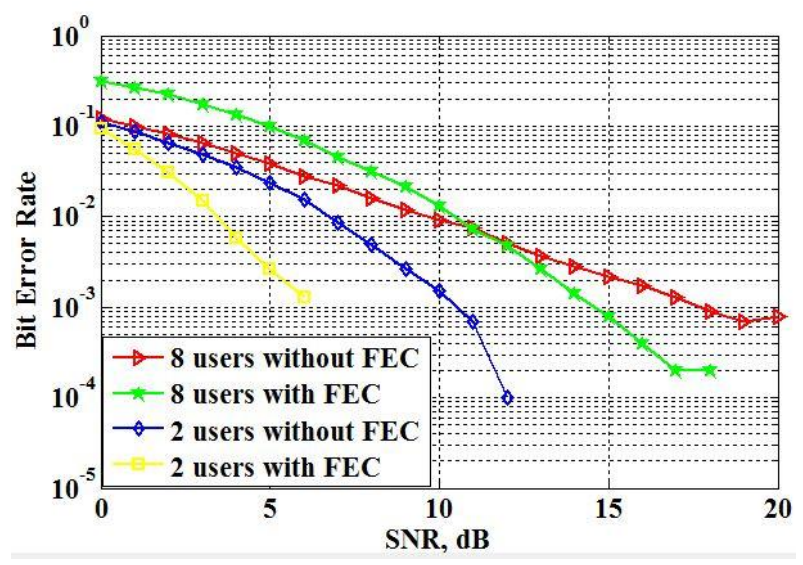

Fig 6: Simulated BER with and without FEC codes using MMSE

\section{CONCLUSION}

Simulation results are given in section 3 and it shows MMSE combining technique provides better SNR performance for a MC-CDMA system to eliminate inter symbol interference as compared to ZF combining technique .Simulation results also shows that the performance of MC-CDMA improves when BPSK is used instead of QPSK as BPSK is a robust scheme in the arena of wireless channels. Also when convolution codes with viterbi decoding are applied as FEC codes along with BPSK and QPSK modulation techniques for ZF and MMSE combining techniques, the performance of MC-CDMA system improves considerably.

\subsection{Future Work}

In future this work can be extended for other mapping schemes like MSK, QAM etc along with FEC coding. Also the performance of MC-CDMA system for other equalization techniques like MRC, EGC etc over Rician , Nakagami or weibull channel models can be evaluated by MATLAB simulations.

\section{REFERENCES}

[1] K. Fazel and S. Kaiser, Multi-Carrier and Spread Spectrum Systems. Second Edition, John Wiley \& Sons

[2] S.Kuzhaloli and K.S.Shaji "Comparison of Equalization Techniques for an MIMO MC-CDMA system" IEEE International Conference on Circuit, Power and Computing Technologies, 2015. 
[3] Kanchana Katta, "Design of Convolutional Encoder and Viterbi Decoder using MATLAB" International Journal For Research In Emerging Science And Technology, vol 1, issue-7, December 2014

[4] Module 6 "Channel coding", version 2 ECE IIT, kharagpur, lesson 35 "Convolutional codes" https://www.coursehero.com/file/7116029/m6135/

[5] Gagandeep Singh Dhaliwal and Navpreet Kaur , "BER based Performance Analysis of MCCDMA over Multipath Channels" International Journal of Computer Applications, vol 69, No.22, May 2013.
[6] Wong \& Lok, Theory of Digital Communications. Chapter-4 Inter symbol Interference and Equalization.

[7] nptel.ac.in/courses/117104099/

[8] John G. Proakis and Masoud Salehi, Communication Systems Engineering. Prentice-Hall, 2002.

[9] Andreas F. Molisch, Wireless Communications. Second Edition Wiley, 2011.

[10] Theodore S. Rappaport, Wireless Communication Principal and Practice. Second Edition, Pearson education, 2002. 